\title{
Potential for Mobile Coverage Improvement in Urban Environments
}

\author{
R C Atkinson and J Dunlop \\ Department of Electronic and Electrical Engineering \\ University of Strathclyde \\ Glasgow G1 1XW, Scotland \\ email : r.atkinson@eee.strath.ac.uk \\ Tel : +44 1415524400 x 2879 Fax : +44 1415524968
}

\begin{abstract}
The migration of UMTS carrier frequencies to the $2 \mathrm{GHz}$ band has stimulated interest in radio coverage in urban environments. This paper examines the problem of poor signal quality close to buildings. It uses simulation based on approximate propagation models in a "generic" urban environment to estimate the relationship between size of coverage black spots and distance from the serving BS. Consideration is also given to the possibility of using channels allocated to in-building picocells as a means of mitigating against coverage black spots.
\end{abstract}

\section{INTRODUCTION}

Many second generation mobile communication systems experience coverage black spots in urban environments, in particular close to shadowing buildings due to either; poor received signal strength (RSS), poor signal quality, or a combination of both. A key requirement of $3^{\text {rd }}$ generation mobile communication systems is continuous coverage between indoor and outdoor environments. The effect of black spots close to buildings presents significant difficulties in providing continuous coverage and has major implications for handover, link adaptation and power control algorithms proposed for third generation systems.

This paper presents the results of a number of simulations based on approximate propagation models to predict the RSS and carrier-tointerference ratio $(\mathrm{C} / \mathrm{I})$ in a generic urban environment at both 900 and $1800 \mathrm{MHz}$. This information being used to analyse the interaction of radio resource management techniques in this environment. This will be incorporated into a work area within the UK Centre of Excellence in Mobile and Personal Communications (MVCE). The results show the area of the black spots close to buildings increases with the distance between mobile and BS.

It is a stated goal of $3^{\text {rd }}$ generation systems to provide a common air interface over as wide a range of operating environments as possible. Under such circumstances dynamic channel assignment between indoor picocells and outdoor macrocells may be contemplated. This paper demonstrates that such techniques could be used to alleviate the radio coverage problem close to buildings. Simulation results have been produced which demonstrate the viability of this option within appropriate constraints.

\section{SIGNAL QUALITY IN URBAN MACRO CELLS}

It has become clear that as carrier frequencies rise coverage in urban districts results in larger areas with poor signal quality. It is well known that higher frequencies are more susceptible to free-space path loss, however this, in itself, does not fully explain the black spots in signal coverage.

The improved Walfisch-Bertoni Urban Propagation Model [1-5] provides a convenient means for estimating sector averaged RSS and $\mathrm{C} / \mathrm{I}$ experienced by mobiles close to shadowing buildings. The model proposes that at 'reasonable' distances from the BS the main propagation paths are over rooftops with a 
diffraction process providing signals at street levels. The convenience of this model is attributed to the term used in calculating the diffraction loss from rooftop down to street level. This term allows the diffraction angle and hence the mobile-building separation distance to be explicitly specified.

A brief explanation of the Walfisch-Bertoni Model follows. The model is composed of three terms; free-space path loss, screen loss, and diffraction loss. The screen loss component models the losses incurred due to propagation over a number of rooftops. The diffraction loss term predicts the losses introduced due to the diffraction process from rooftop down to street level.

The model approximates the urban environment as a series of parallel cuboids (representing rows of buildings between base and mobile). For simplicity, it is assumed that one set of building-rows is oriented in a north/south direction, due north being represented as a bearing of $0^{\circ}$. The BS in figure 1 may thus be specified in terms of a bearing $\phi$ and distance relative to the mobile. The bearing can be used to calculate the street orientation angle - an important parameter in calculating screen loss.

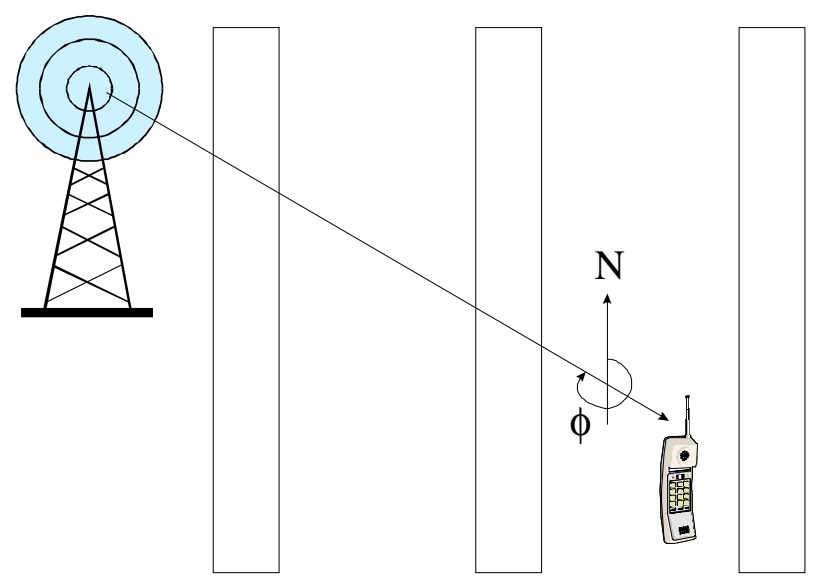

Figure 1 : Non-line-of-sight propagation path

The model may be extended to accommodate a number of different interfering BSs at other bearings and distances from the mobile. The signals from the interfering BSs will reach the mobile by similar paths to the one shown in figure 1, the RSS from the individual interfering
BSs being summed to produce the total interference term. To accommodate the effects from shadowing and screen loss from the various interfering BSs, an east/west orientation of streets is introduced and hence another row of cuboids (buildings) is superimposed on the original north/south orientation. Whilst not being a strictly practical model, the layout of figure 2 does allow for changes in shadowing as the mobile moves amongst the surrounding buildings and is a useful 'generic' model of the urban environment.

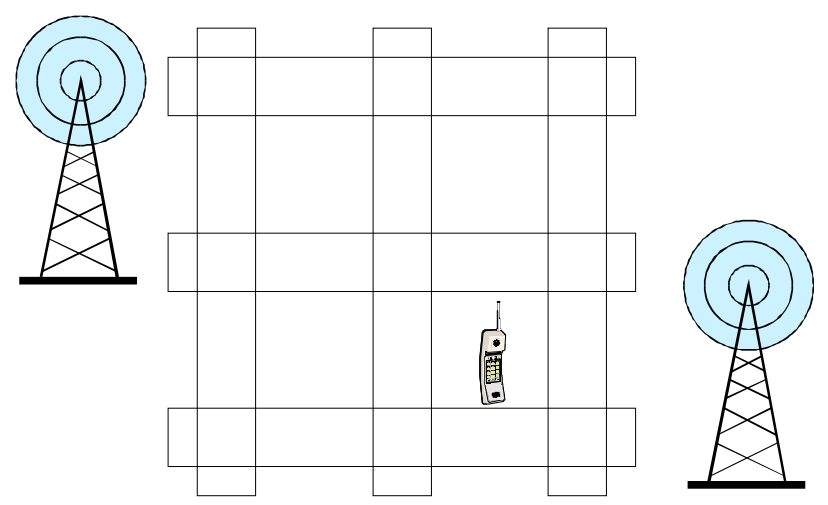

Figure 2 : Approximate urban layout

The mobile in figure 2 is now effectively surrounded by buildings and this specific circumstance can be used to illustrate a nonline-of-sight situation in which propagation takes place over rooftops.

A number of simulations were conducted using the Walfisch-Bertoni model to predict RSS and $\mathrm{C} / \mathrm{I}$ across an urban street at various distances from the BS. This paper will consider three sample distances; $500 \mathrm{~m}, 750 \mathrm{~m}$, and $1 \mathrm{~km}$. The building heights are representative of those in typical dense city centres. The following parameters are assumed throughout; BS antenna height $=40 \mathrm{~m}$, mobile antenna height $=2 \mathrm{~m}$, mean building height $=20 \mathrm{~m}$, mean building separation $=50 \mathrm{~m}$, street width $=30 \mathrm{~m}$, and BS transmitter power $=46 \mathrm{dBm}$. It is also assumed that the BS transmits continuously on full power, and all antennas are regarded as isotropic. The parameters used in the simulations assume a radius $R=1 \mathrm{~km}$ (small-cell) and a cluster size $k$ $=4$ (typical for urban environments). Based on the bearing definition of figure 1 , it is assumed the serving $\mathrm{BS}$ is at a bearing of $270^{\circ}$ relative to 
the mobile (i.e. due west) and is surrounded by six co-channel BSs at bearings separated by $60^{\circ}$ with respect to the serving BS. The distance $D$ between the serving and co-channel $\mathrm{BSs}$ is given by:

$$
D=\sqrt{3 k} R
$$

The results of the simulations to predict $\mathrm{C} / \mathrm{I}$ in urban streets at frequencies of $900 \mathrm{MHz}$ and 1.8 $\mathrm{GHz}$ are presented in figures 3,4 and 5. A $9 \mathrm{~dB}$ threshold commonly used as a minimum $\mathrm{C} / \mathrm{I}$ ratio for acceptable performance in GSM systems is shown on the plots.

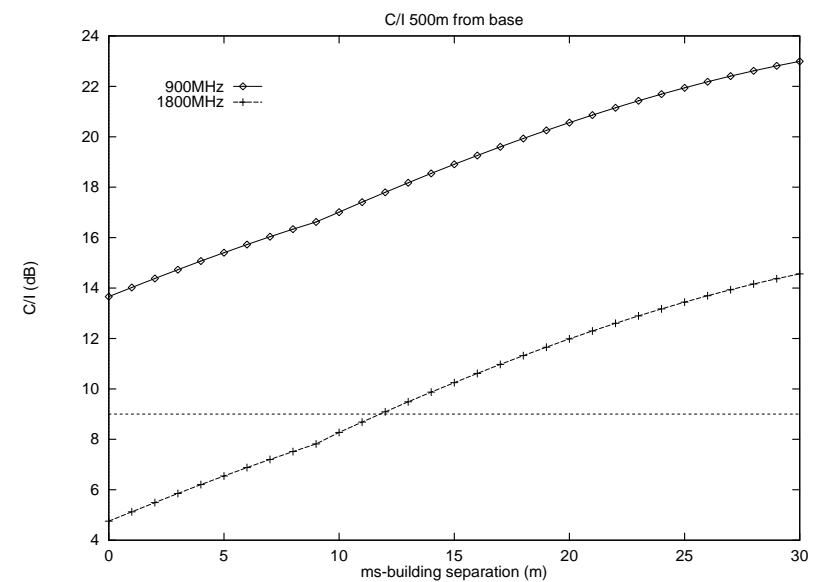

Figure 3 : C/I at 500m from BS

At $500 \mathrm{~m}$ from the BS the model predicts that the $\mathrm{C} / \mathrm{I}$ at $900 \mathrm{MHz}$ is always above the threshold, irrespective of the distance between the mobile and the shadowing building. However, at 1800 $\mathrm{MHz}$ the model predicts that the mobile must be at least $11 \mathrm{~m}$ away from the shadowing building to achieve a $\mathrm{C} / \mathrm{I}$ greater than $9 \mathrm{~dB}$. This figure of $11 \mathrm{~m}$ represent the size of the coverage black spot at this frequency.

At $750 \mathrm{~m}$ from the $\mathrm{BS}$, in the $900 \mathrm{MHz}$ band, the $\mathrm{C} / \mathrm{I}$ does not cross the $9 \mathrm{~dB}$ threshold until the mobile is $4 \mathrm{~m}$ from the building. At $1800 \mathrm{MHz}$ this distance increases to $23 \mathrm{~m}$. It should be noted that the predicted, $\mathrm{C} / \mathrm{I}$ increases by $9.9 \mathrm{~dB}$, at $900 \mathrm{MHz}$ and by $10.5 \mathrm{~dB}$ at $1800 \mathrm{MHz}$, as the mobile crosses the street.

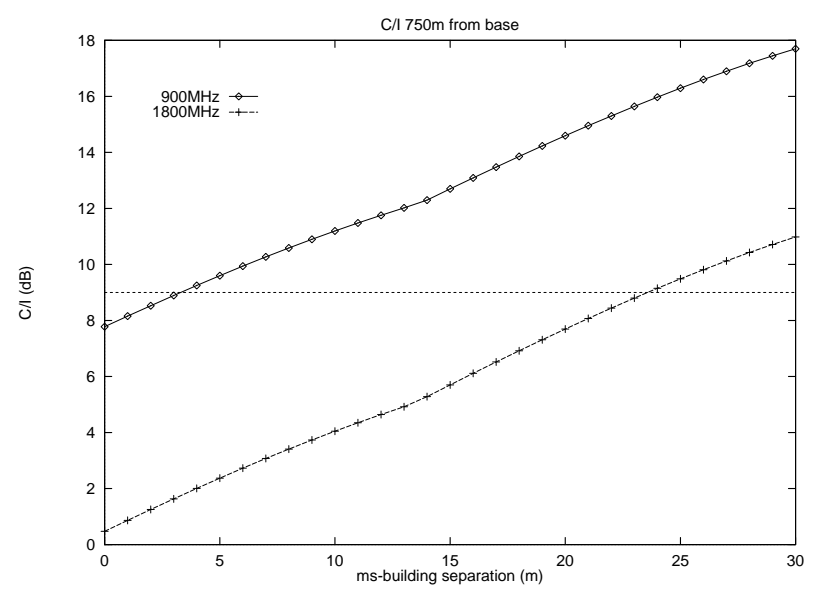

Figure 4 : C/I at 750m from BS

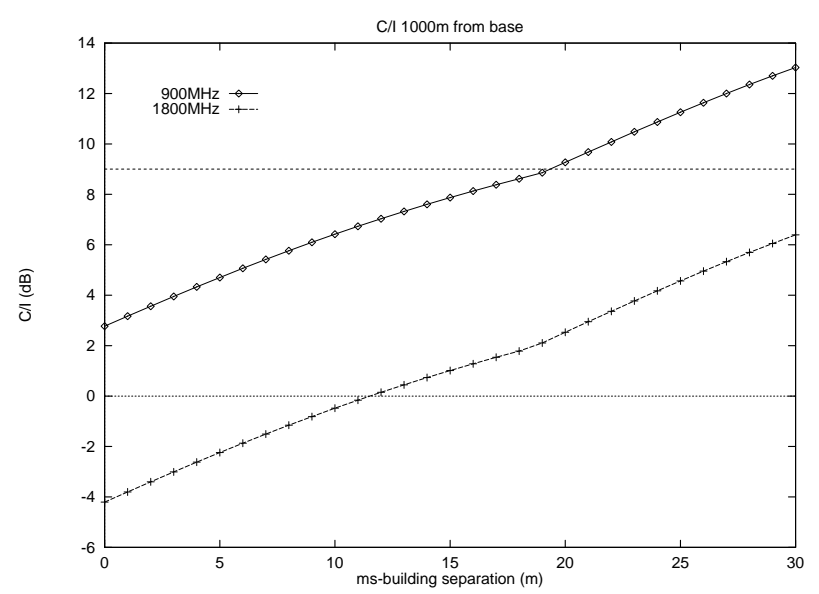

Figure 5 : $\mathrm{C} / \mathrm{I}$ at $1 \mathrm{~km}$ from $\mathrm{BS}$

At $1 \mathrm{~km}$ from the $\mathrm{BS}$, for $900 \mathrm{MHz}$, the $\mathrm{C} / \mathrm{I}$ is below the $9 \mathrm{~dB}$ threshold until the mobile is $20 \mathrm{~m}$ from the building. Since the width of the street is $30 \mathrm{~m}$ this requires the mobile to be located on the opposite side of the street from the main shadower (building shadowing the serving BS) for adequate reception. At $1800 \mathrm{MHz}$ the $\mathrm{C} / \mathrm{I}$ is always below the 9dB-threshold i.e. adequate signal quality is never attained. The $\mathrm{C} / \mathrm{I}$ increases by $10.2 \mathrm{~dB}$ for $900 \mathrm{MHz}$ and $10.6 \mathrm{~dB}$ from $1800 \mathrm{MHz}$, at $1 \mathrm{~km}$ from the $\mathrm{BS}$ as the mobile crosses the street.

It can therefore be concluded that the increase in $\mathrm{C} / \mathrm{I}$ as the mobile crosses the street is dependant on two factors; the mobile's range from the BS and the carrier frequency. It is evident from the plots of $\mathrm{C} / \mathrm{I}$ that the size of the black spots increases with the mobiles distance from the BS. 


\section{EXTERNAL COVERAGE FROM INTERNAL BSS}

One possible solution for increasing C/I close to buildings is to utilise the capacity of in-building BSs. In many circumstances, the RSS from a nearby internal BS is higher than that from a distant external BS. It has been shown that [6] thick exterior walls can contribute as little as 15 $\mathrm{dB}$ to losses in signal strength.

Simulations were conducted using the Motley Indoor Propagation Model [6] to predict the strength of signals outside a building from a picocellular base station located within the building. The results obtained were combined with those collected for RSS in the same location from an urban macrocellular BS. The comparison was based on the assumption that the picocellular BS emits a constant power of $24 \mathrm{dBm}$ and the intervening wall was composed of concrete of thickness $250 \mathrm{~mm}$. It was further assumed that the picocellular BS is located at the back of a building of width $30 \mathrm{~m}$.

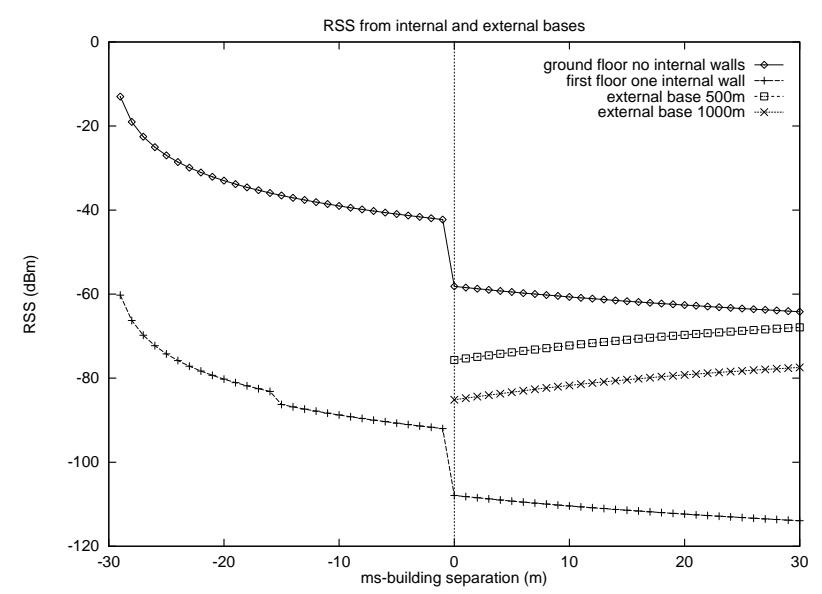

\section{Figure 6 : RSS from internal and external BSs}

Figure 6 indicates the predicted RSS from the picocellular BS, when located on the ground floor, is greater than that of urban macrocellular BSs located at both $500 \mathrm{~m}$ and $1 \mathrm{~km}$ from the mobile. When the picocellular BS is located on the second floor, the RSS in the street is significantly reduced, and is less than that from the urban macrocellular BSs at both ranges. Thus an internal BS, located on the ground floor may superior signal strength to a mobile located in an adjacent street than an external BS located over 500m away.

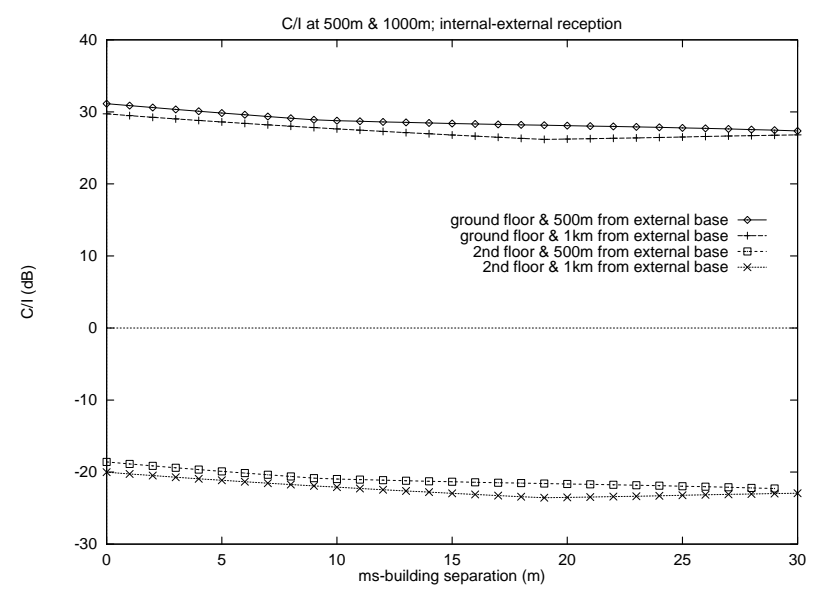

Figure 7 : C/I from internal and external BSs

If the choice of carrier frequency on the internal BS is not reused by any other nearby internal $\mathrm{BS}$, the only co-channel interference at the mobile will be due to emissions from other external BSs. Thus, C/I was calculated using RSS from the internal BS and the interference from surrounding external BS. It is assumed that the nearest macrocellular BS is aware of the existence of the internal picocellular BS and refrains from transmitting on it's carrier frequencies. The nearest $\mathrm{BS}$ was therefore excluded when calculating the interference term. Figure 7 demonstrates that when the internal BS is located on the ground floor, $\mathrm{C} / \mathrm{I}$ is much greater than the $9 \mathrm{~dB}$ threshold at both $500 \mathrm{~m}$ and $1 \mathrm{~km}$. When the internal BS is located on the second floor, $\mathrm{C} / \mathrm{I}$ is significantly below the threshold at both ranges.

The improvement in RSS has the effect of decreasing the size of the coverage black spots and effectively improves the coverage close to urban buildings.

\section{CONCLUSIONS}

The improvement in RSS and hence C/I close to shadowing buildings using internal picocellular BSs significantly reduces the coverage black spots experienced by current second generation systems. Furthermore, this solution demonstrates that continuous indoor-outdoorcoverage, a key requirement for future generations of mobile systems, can be provided 
by locating the in-building $\mathrm{BS}$ on the ground floor.

The concept of utilising the capacity of picocellular BSs to provide additional coverage is a viable option with appropriate provision for dynamic channel assignment between the indoor and outdoor radio interfaces.

As can be seen from the plots of $\mathrm{C} / \mathrm{I}$, mobile terminals located in the centre of the street generally receive adequate mean signal quality, while those closest to the main shadowing building, i.e. on the near side pavement (pedestrians) are more likely to encounter black spots. Thus, it is not anticipated that handover algorithms need concern themselves with handing over a large number of fast moving mobiles from internal BS to internal BS.

In addition to providing macro cellular coverage to a number of mobiles, the external BS could provide a number of trunked links to internal BSs which in turn would provide superior coverage. The external BS supplying the trunked link to the internal BS would be the one with the lowest path loss, this BS would require knowledge of the frequencies in use (radiated into streets) by the internal BS in order to implement a dynamic channel allocation strategy. This is a topic of further study.

\section{ACKNOWLEDGEMENTS}

The authors acknowledge the support of the UK Virtual Centre of Excellence in Mobile and Personal Communications.

\section{REFERENCES}

1. Walfisch $\mathrm{J}$ and Bertoni HL, A Theoretical Model of UHF Propagation in Urban Environments, IEEE Trans. Antennas and Propagation, Vol. 36, No 12, Dec 1988, pp. 1788-1796.

2. Bertoni HL, Honcharenko W, Maciel LR and $\mathrm{Xia} \mathrm{HH}, \mathrm{UHF}$ Propagation Prediction for Wireless Personal Communications, Proc. IEEE Vol. 82, No 9, Sept. 1994.
3. Xia HH, An Analytical Model for Predicting Pathloss in Urban and Suburban Environments, Proceedings of PIMRC '96, Taipei, Taiwan, Oct 1996, pp19-23.

4. Cardona N, Moller P and Alonso F, Applicability of Walfisch-type urban propagation models, Electronics Letters, Vol 31, No 23, November 1995, p 1971-1972.

5. Cardona Marcet N, Navarro Mas F and Moller P, Applicability of Walfisch-type urban propagation models, COST 231 TD(94) 134, Darmstadt, September 1994

6. Gollreiter R (Ed.), Channel Models Issue 2, Advanced TDMA Mobile Access, R2084/ESG/CC3/DS/P/029/b1, 1994, pp. 7781 . 